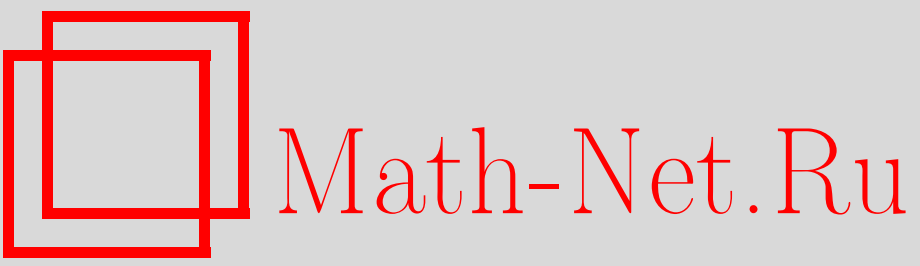

М. В. Карасёв, Е. М. Новикова, Алгебра с полиномиальными коммутационными соотношениями для эффекта Зеемана в поле Кулона-Дирака, ТМФ, 2005, том 142, номер 1, 127-147

DOI: https://doi.org/10.4213/tmf1762

Использование Общероссийского математического портала Math-Net.Ru подразумевает, что вы прочитали и согласны с пользовательским соглашением

http://www. mathnet.ru/rus/agreement

Параметры загрузки:

IP: 54.80 .97 .219

26 апреля 2023 г., 18:25:03 
ТЕОРЕТИЧЕСКАЯ

И МАТЕМАТИЧЕСКАЯ

ФИЗИКА

Том 142, № 1

январь, 2005

(C) 2005 r.

М.В. Карасев*, Е.М. Новикова*

\section{АЛГЕБРА С ПОЛИНОМИАЛЬНЫМИ КОММУТАЦИОННЫМИ СООТНОШЕНИЯМИ ДЛЯ ЭФФЕКТА ЗЕЕМАНА В ПОЛЕ КУЛОНА-ДИРАКА}

Рассматривается модель движения частицы в поле электромагнитного монополя (поле Кулона-Дирака), возмущенном однородным магнитным и неоднородным электрическим полями. После квантового усреднения возникает интегрируемая система, гамильтониан которой выражается через образующие алгебры с полиномиальными коммутационными соотношениями. Строятся неприводимые представления этой алгебры, а также ее гипергеометрические когерентные состояния. С помощью этих состояний получено представление собственных функций исходной задачи через решения модельного обыкновенного дифференциального уравнения и указана асимптотика собственных значений в главном порядке теории возмущений, где полностью снимается вырождение спектра.

Ключевые слова: интегрируемые системы, монополь Дирака, нелинейные коммутационные соотношения, когерентные состояния, спектральные асимптотики.

\section{1. ВВЕДЕНИЕ}

Эта статья - вторая из цикла работ, посвяшенных 30-летию выхода в свет книги В. П. Маслова "Операторные методы" [1], послужившей отправной точкой многих современных исследований в области некоммутативного анализа и квантовой геометрии.

Первая статья данного цикла [2] содержит подробное введение, где в контексте задачи о квантовой частице в электромагнитном поле обсуждается некоторый общий механизм возникновения алгебр операторов с нелинейными коммутационными соотношениями, а также даны ссылки на литературу и подробно описывается используемая здесь техника. В статье [2] рассмотрена также конкретная спектральная задача для частищы, движущейся в поле электромагнитного монополя (поле Кулона-Дирака), возмушенном аксиальным электрическим полем. Показано, что в такой модели возникает алгебра с квадратичными коммутационными соотношениями, обобщающая алгебру, найденную в работе [3] для эффекта Зеемана в атоме водорода (см. также [4]).

\footnotetext{
* Московский государственный институт электроники и математики, Москва, Россия. E-mail: karasev@miem.edu.ru; novikova@miem.edu.ru
} 
В данной работе рассматривается другая конфигурация: поле Кулона-Дирака, возмушенное однородным магнитным и неоднородным электрическим полями. Исследуется случай, когда напряженность возмушаюшего электрического поля (в безразмерных единицах) является величиной второго порядка малости по сравнению с напряженностью возмушающего магнитного поля. В такой системе после усреднения появляется $a \Omega$ гебра с полиномиальным.м комммтационныцми соотношениями, структура которых универсальна и определяется лишь арифметической пропоршией между главными квантовыми числами. Неприводимые представления и когерентные состояния этой алгебры позволяют найти (приближенно) спектр и собственные функции исходной неинтегрируемой системы.

Работа имеет следующую структуру. В начале раздела 2 гамильтониан системы приводится к виду "действие" + возмущение. Далее, после квантового усреднения возникает новый оператор возмушения, коммутируюший с “действием". В свою очередь, это усредненное возмущение тоже представляет собой сумму некоторого оператора типа "действие" и возмушаюшего оператора второго порядка малости. К этой сумме можно еше раз применить квантовое усреднение. В результате исходная спектральная задача сводится к спектральной задаче для трех коммутируюших операторов: два "действия" +дважды усредненное возмущение.

Основная часть второго раздела посвящена исследованию дважды усредненного возмушения. Этот оператор является элементом алгебры $\mathcal{F}_{\text {quant }}$ совместных квантовых интегралов движения двух коммутирующих операторов “действие". В п. 2.6 выписаны генераторы этой алгебры и полиномиальные коммутационные соотношения, которым они подчинены. Кроме того, в модельном примере (с простейшим потеншиалом возмущающего электрического поля) получено явное выражение для дважды усредненного гамильтониана в виде функции от генераторов алгебры $\mathcal{F}_{\text {quant }}$.

Выделенная алгебра с полиномиальными коммутационными соотношениями детально исследуется в разделе 3 . Строятся ее неприводимые представления обыкновенными дифференциальными операторами в пространстве полиномов; выписываются соответствующие когерентные состояния и определяется когерентное преобразование, сплетаюшее абстрактное представление данной алгебры с неприводимым.

В разделе 4 с помошью когерентного преобразования спектральная задача для дважды усредненного гамильтониана сводится к обыкновенному дифференциальному уравнению в гильбертовом пространстве полиномов. В модельном примере это дифференциальное уравнение имеет второй или третий порядок.

В результате вычисляются собственные значения исходного гамильтониана, а также соответствуюшие им собственные функции в главном приближении теории возмушений, где полностью снимается вырождение старшей части спектра.

В приложении приведена реализация задачи в гильбертовом пространстве $L^{2}\left(\mathbb{R}^{4}\right)$, выписаны формулы для генераторов алгебры $\mathcal{F}_{\text {quant }}$ и формулы для соответствующих когерентных состояний в $L^{2}\left(\mathbb{R}^{4}\right)$. 


\section{2. ДВОЙНОЕ КВАНТОВОЕ УСРЕДНЕНИЕ}

Предположим, что в некотором гильбертовом пространстве $\mathcal{L}$ операторы $\mathbf{q}=$ $\left(\mathbf{q}_{1}, \mathbf{q}_{2}, \mathbf{q}_{3}\right)$ и $\mathbf{p}=\left(\mathbf{p}_{1}, \mathbf{p}_{2}, \mathbf{p}_{3}\right)$ задают неприводимое представление алгебры

$$
\begin{aligned}
{\left[\mathbf{q}_{j}, \mathbf{q}_{k}\right]=0, \quad\left[\mathbf{p}_{j}, \mathbf{q}_{k}\right] } & =-i \hbar \delta_{j k}, \quad\left[\mathbf{p}_{j}, \mathbf{p}_{k}\right]=i \hbar \mu \varepsilon_{j k l} \frac{\mathbf{q}_{l}}{|\mathbf{q}|^{3}}, \\
\mathbf{q}_{j} & =\mathbf{q}_{j}^{\star}, \quad \mathbf{p}_{j}=\mathbf{p}_{j}^{\star},
\end{aligned}
$$

где $\hbar>0$, звездочка обозначает операторное сопряжение, а константа $\mu$ (магнитный заряд) подчинена условию квантования: $\mu=\hbar k / 2, k \in \mathbb{Z}$. Число $k$ раз и навсегда фиксировано. Алгебра (2.1) используется для описания монополя Дирака [5]. Ее реализация операторами в $L^{2}\left(\mathbb{R}^{4}\right)$, обсуждение и ссылки на литературу приведены в работе [2]. Квантовая система, которая исследуется в данной работе, - это частица в поле Кулона-Дирака, возмущенном однородным магнитным и неоднородным әлектрическим полями. Ее гамильтониан имеет вид

$$
\left(\mathbf{p}_{1}-\frac{\varepsilon}{2} \mathbf{q}_{2}\right)^{2}+\left(\mathbf{p}_{2}+\frac{\varepsilon}{2} \mathbf{q}_{1}\right)^{2}+\mathbf{p}_{3}^{2}-\frac{1}{|\mathbf{q}|}+\frac{\mu^{2}}{|\mathbf{q}|^{2}}+\varepsilon^{2} \mathcal{U}(\mathbf{q})
$$

в гильбертовом пространстве $\mathcal{L}$ представления алгебры (2.1). Здесь однородное магнитное поле предполагается направленным вдоль третьей оси, $\mathcal{U}(\mathbf{q})$ - потенциал электрического поля, а $\varepsilon$ - параметр, характеризуюший величину напряженности полей. Мы предполагаем, что $\varepsilon \ll 1$.

Для наглядности наряду со случаем общего неоднородного потенциала $\mathcal{U}$ будем рассматривать также модельный пример, когда

$$
\mathcal{U}(q)=\gamma q_{1} q_{2}^{2},
$$

где $\gamma=$ const.

2.1. Сведение задачи к виду "действие" +возмущение. Наша исходная спектральная задача имеет вид

$$
\left(\mathbf{H}_{0}+\varepsilon\left(\mathbf{q}_{1} \mathbf{p}_{2}-\mathbf{q}_{2} \mathbf{p}_{1}\right)+\varepsilon^{2}\left(\frac{1}{4}\left(\mathbf{q}_{1}^{2}+\mathbf{q}_{2}^{2}\right)+\mathcal{U}(\mathbf{q})\right)\right) \psi=\mathcal{E} \psi, \quad \psi \in \mathcal{L},
$$

где

$$
\mathbf{H}_{0}=|\mathbf{p}|^{2}-\frac{1}{|\mathbf{q}|}+\frac{\mu^{2}}{|\mathbf{q}|^{2}}
$$

- гамильтониан частишы в поле Кулона-Дирака (все подробности о свойствах $\mathbf{H}_{0}$ и ссылки на литературу см. в работе [2]). Дискретный спектр лежит на отришательной полуоси $\mathcal{E}<0$, поэтому мы полагаем

$$
\mathcal{E}=-\frac{1}{4 \lambda^{2}}, \quad \lambda>0
$$

5 Теоретическая и математическая физика, т. 142, № 1, 2005 г. 
После умножения на $\lambda|\mathbf{q}|$ сведем (2.3) к задаче

$$
\left(\mathbf{S}_{0}+\varepsilon \mathbf{X}_{1}+\varepsilon^{2} \mathbf{Y}_{1}\right) \psi=\lambda \psi, \quad \psi \in \mathcal{L}_{-},
$$

в гильбертовом пространстве $\mathcal{L}_{-}$со скалярным произведением

$$
\left\langle\psi_{1}, \psi_{2}\right\rangle_{-} \stackrel{\text { def }}{=} \frac{\pi}{4}\left\langle\frac{1}{|\mathbf{q}|} \psi_{1}, \psi_{2}\right\rangle
$$

где $\langle\cdot, \cdot\rangle$ - скалярное произведение в $\mathcal{L}$. Здесь $\mathbf{S}_{0}$ - оператор “действие” (см. [2]):

$$
\mathbf{S}_{0} \stackrel{\text { def }}{=} s(\lambda, \stackrel{2}{\mathbf{q}}, \stackrel{1}{\mathbf{p}}), \quad s(\lambda, q, p) \stackrel{\text { def }}{=} \lambda|q||p|^{2}+\frac{\mu^{2} \lambda}{|q|}+\frac{|q|}{4 \lambda}
$$

a $\mathbf{X}_{1}$ и $\mathbf{Y}_{1}$ задаются формулами

$$
\mathbf{X}_{1}=\lambda|\mathbf{q}|\left(\mathbf{q}_{1} \mathbf{p}_{2}-\mathbf{q}_{2} \mathbf{p}_{1}\right), \quad \mathbf{Y}_{1}=\lambda|\mathbf{q}|\left(\frac{1}{4}\left(\mathbf{q}_{1}^{2}+\mathbf{q}_{2}^{2}\right)+\mathcal{U}(\mathbf{q})\right)
$$

2.2. Квантовое усреднение. Схема квантового усреднения, которую мы используем, описана в работах [2], [4]. Основные расчетные формулы следуюшие. Для каждого оператора $\mathbf{F}$ определим операторы

$\underline{\mathbf{F}}=\frac{1}{2 \pi} \int_{0}^{2 \pi} e^{-(i \tau / \hbar) \mathbf{S}_{0}} \mathbf{F} e^{(i \tau / \hbar) \mathbf{S}_{0}} d \tau, \quad \mathbf{F}^{\#}=\frac{1}{2 \pi} \int_{0}^{2 \pi}(\pi-\tau) e^{-(i \tau / \hbar) \mathbf{S}_{0}} \mathbf{F} e^{(i \tau / \hbar) \mathbf{S}_{0}} d \tau$.

Используя эти обозначения, построим усредняющий оператор

$$
\mathbf{U}=\exp \left\{-\frac{i \varepsilon}{\hbar}\left(\mathbf{S}_{1}^{\#}+\varepsilon \mathbf{S}_{2}^{\#}\right)\right\}, \quad \text { где } \mathbf{S}_{2} \stackrel{\text { def }}{=} \frac{i}{2 \hbar}\left[\mathbf{S}_{1}^{\#}, \mathbf{S}_{1}+\underline{\mathbf{S}_{1}}\right] .
$$

Тогда имеют место тождества

$$
\mathbf{U}^{-1}\left(\mathbf{S}_{0}+\varepsilon \mathbf{S}_{1}\right) \mathbf{U}=\mathbf{S}_{0}+\varepsilon \underline{\mathbf{S}_{1}}+\varepsilon^{2} \underline{\mathbf{S}_{2}}+O\left(\varepsilon^{3}\right), \quad\left[\underline{\mathbf{S}_{1}}, \mathbf{S}_{0}\right]=\left[\underline{\mathbf{S}_{2}}, \mathbf{S}_{0}\right]=0 .
$$

Таким образом, в результате квантового усреднения получены новые гамильтонианы $\underline{\mathbf{S}_{1}}$ и $\underline{\mathbf{S}_{2}}$, коммутируюшие со старшим членом $\mathbf{S}_{0}$.

2.3. Усредненный оператор. Вычислим усредненное возмушение $\underline{\mathbf{S}_{1}}+\varepsilon \underline{\mathbf{S}_{2}}$ для нашей задачи, в которой $\mathbf{S}_{1}=\mathbf{X}_{1}+\varepsilon \mathbf{Y}_{1}$. 
ПРЕДЛОЖЕНИЕ 2.1. Пусть $\mathbf{S}_{0}$ - оператор “действие" (2.5), a $\mathbf{X}_{1}, \mathbf{Y}_{1}$ - операторы (2.6). Тогда выполнень тождества

$$
\begin{gathered}
\mathbf{U}^{-1}\left(\mathbf{S}_{0}+\varepsilon \mathbf{X}_{1}+\varepsilon^{2} \mathbf{Y}_{1}\right) \mathbf{U}=\mathbf{S}_{0}+\varepsilon \underline{\mathbf{X}_{1}}+\varepsilon^{2}\left(\underline{\mathbf{X}_{2}}+\underline{\mathbf{Y}_{1}}\right)+O\left(\varepsilon^{3}\right), \\
{\left[\underline{\mathbf{X}_{1}}, \mathbf{S}_{0}\right]=\left[\underline{\mathbf{X}_{2}}, \mathbf{S}_{0}\right]=\left[\underline{\mathbf{Y}_{1}}, \mathbf{S}_{0}\right]=0,}
\end{gathered}
$$

в которых усредненное возмущение задается гамильтонианами

$$
\begin{aligned}
\underline{\mathbf{X}_{1}}= & 2 \lambda^{2}\left(\left(\mathbf{S}_{0}+\mu\right) \mathbf{J}_{3}+\left(\mathbf{S}_{0}-\mu\right) \mathbf{K}_{3}\right), \\
\underline{\mathbf{X}_{2}}=- & -2 \lambda^{4}\left(\mathbf{S}_{0}\left(\mathbf{J}_{3}+\mathbf{K}_{3}\right)^{2}+\mu^{2} \mathbf{S}_{0}+2 \mu\left(\mathbf{J}_{3}^{2}-\mathbf{K}_{3}^{2}\right)\right), \\
\underline{\mathbf{Y}_{1}}= & \lambda^{4}\left(3 \mathbf{S}_{0}^{3}-\mu^{2} \mathbf{S}_{0}-4 \mathbf{S}_{0}\left(\mathbf{J}_{3}^{2}+\mathbf{K}_{3}^{2}\right)-2 \mu\left(\mathbf{J}_{3}^{2}-\mathbf{K}_{3}^{2}\right)+4 \mathbf{S}_{0} \mathbf{J}_{3} \mathbf{K}_{3}-\right. \\
& \left.\quad-8 \mathbf{S}_{0}\left(\mathbf{J}_{1} \mathbf{K}_{1}+\mathbf{J}_{2} \mathbf{K}_{2}\right)+\hbar^{2} \mathbf{S}_{0}\right)+\lambda \underline{\mathbf{q} \mid \mathcal{U}(\mathbf{q})},
\end{aligned}
$$

а оператор $\underline{|\mathbf{q}| \mathcal{U}(\mathbf{q})}$ (последнее слагаемое в $\left.\underline{\mathbf{Y}_{1}}\right)$ - формулой

$$
\underline{|\mathbf{q}| \mathcal{U}(\mathbf{q})}=\frac{\lambda}{2 \pi} \int_{0}^{2 \pi}\left(2 \mathbf{S}_{0}+\mathbf{v} e^{i \tau}+\mathbf{v}^{*} e^{-i \tau}\right) \mathcal{U}\left(\lambda\left(2(\mathbf{J}-\mathbf{K})+\mathbf{V} e^{i \tau}+\mathbf{V}^{*} e^{-i \tau}\right)\right) d \tau
$$

В модельном примере (2.2) имеем

$$
\begin{aligned}
\underline{\underline{\mathbf{q} \mid \mathbf{q}_{1} \mathbf{q}_{2}^{2}}=\lambda^{4}} & \left(2 0 \mathbf { S } _ { 0 } \left(2 \mathbf{J}_{1} \mathbf{J}_{2}^{2}-2 \mathbf{K}_{1} \mathbf{K}_{2}^{2}-7 \mathbf{J}_{1} \mathbf{J}_{2} \mathbf{K}_{2}+7 \mathbf{J}_{2} \mathbf{K}_{1} \mathbf{K}_{2}-5 \mathbf{J}_{2}^{2} \mathbf{K}_{1}+5 \mathbf{J}_{1} \mathbf{K}_{2}^{2}-\right.\right. \\
& \left.-\mathbf{J}_{3}^{2} \mathbf{K}_{1}+\mathbf{J}_{1} \mathbf{K}_{3}^{2}+\mathbf{J}_{3} \mathbf{J}_{1} \mathbf{K}_{3}-\mathbf{J}_{3} \mathbf{K}_{3} \mathbf{K}_{1}\right)-8 \mu \mathbf{S}_{0}^{2}\left(\mathbf{J}_{1}+\mathbf{K}_{1}\right)- \\
& -8 \mu^{2} \mathbf{S}_{0}\left(\mathbf{J}_{1}-\mathbf{K}_{1}\right)+4 \mu\left(6 \mathbf{J}_{1} \mathbf{J}_{2}^{2}+6 \mathbf{K}_{1} \mathbf{K}_{2}^{2}-7 \mathbf{J}_{1} \mathbf{J}_{2} \mathbf{K}_{2}-7 \mathbf{J}_{2} \mathbf{K}_{1} \mathbf{K}_{2}-\right. \\
& \left.-5 \mathbf{J}_{2}^{2} \mathbf{K}_{1}-5 \mathbf{J}_{1} \mathbf{K}_{2}^{2}-\mathbf{J}_{3}^{2} \mathbf{K}_{1}-\mathbf{J}_{1} \mathbf{K}_{3}^{2}+\mathbf{J}_{3} \mathbf{J}_{1} \mathbf{K}_{3}+\mathbf{J}_{3} \mathbf{K}_{3} \mathbf{K}_{1}\right)- \\
& -40 i \hbar \mathbf{S}_{0}\left(\mathbf{J}_{2} \mathbf{J}_{3}-\mathbf{K}_{2} \mathbf{K}_{3}+2 \mathbf{J}_{2} \mathbf{K}_{3}-2 \mathbf{J}_{3} \mathbf{K}_{2}\right)- \\
& \left.-24 i \hbar \mu\left(\mathbf{J}_{2} \mathbf{J}_{3}+\mathbf{K}_{2} \mathbf{K}_{3}\right)+12 i \hbar \mu\left(\mathbf{J}_{2} \mathbf{K}_{3}+\mathbf{J}_{3} \mathbf{K}_{2}\right)-12 \hbar^{2} \mu\left(\mathbf{J}_{1}+\mathbf{K}_{1}\right)\right) .
\end{aligned}
$$

Образующие $\mathbf{J}, \mathbf{K}$ алгебры квантовых интегралов движения оператора "действие" и операторы $\mathbf{v}, \mathbf{V}, \mathbf{v}^{*}, \mathbf{V}^{*}$ определень в статье [2] (см. формуль (2.6), (2.7) $u(2.8),(2.9))$.

Усредняющее преобразование $\mathbf{U}$ в первом из тождеств (2.7) имеет вид

$$
\mathbf{U}=\exp \left\{-\frac{i \varepsilon}{\hbar}\left(\mathbf{X}_{1}^{\#}+\varepsilon\left(\mathbf{X}_{2}^{\#}+\mathbf{Y}_{1}^{\#}\right)\right)\right\}
$$

2.4. Второй оператор “действие". Оператор “действие” $\mathbf{S}_{0}$ имеет собственные значения $\lambda_{n}=\hbar(n+1+|k| / 2)$ и собственные подпространства $\mathcal{L}_{-}[n] \subset \mathcal{L}_{-}, n \in \mathbb{Z}_{+}$. Описание этих подпространств дано в работе [2].

Обозначим через $l$ и $r$ взаимно простые натуральные числа такие, что

$$
\frac{n+1+|k| / 2+k / 2}{n+1+|k| / 2-k / 2}=\frac{l}{r}
$$


(в случае $k=0$ нужно положить $l=r=1$ ). Тогда можно записать

$$
n+1+\frac{|k|}{2}+\frac{k}{2}=l d, \quad n+1+\frac{|k|}{2}-\frac{k}{2}=r d,
$$

где $d$ - некоторое натуральное число.

Введем операторы

$$
\mathbf{G}_{0}=l \mathbf{J}_{3}+r \mathbf{K}_{3}, \quad \mathbf{G}_{1}=\frac{1}{2 \lambda^{2} d \hbar}\left(\underline{\mathbf{X}_{2}}+\underline{\mathbf{Y}_{1}}\right) .
$$

Отметим, что спектр $\mathbf{G}_{0}$ на подпространстве $\mathcal{L}_{-}[n]$ состоит из чисел

$$
\hbar\left(r j_{2}-l j_{1}-\frac{l-r}{2}\right)
$$

где $0 \leqslant j_{1} \leqslant r d-1,0 \leqslant j_{2} \leqslant l d-1$. Соответствуюшими собственными функциями являются функции $\psi_{j_{1}, j_{2}}^{n}$, определенные в статье [2] формулой (2.11).

Таким образом, $\mathbf{G}_{0}$ может рассматриваться как новый оператор “действие".

Усредненный возмушаюший оператор в правой части $(2.7)$ на подпространстве $\mathcal{L}_{-}[n]$ принимает вид

$$
\left.\left[\varepsilon \underline{\mathbf{X}_{1}}+\varepsilon^{2}\left(\underline{\mathbf{X}_{2}}+\underline{\mathbf{Y}_{1}}\right)\right]\right|_{\mathcal{L}_{-}[n]}=\varepsilon 2 \hbar d \lambda^{2}\left(\mathbf{G}_{0}+\varepsilon \mathbf{G}_{1}\right) .
$$

2.5. Вторичное квантовое усреднение. К сумме операторов $\mathbf{G}_{0}+\varepsilon \mathbf{G}_{1}$ на $\mathcal{L}_{-}[n]$ можно снова применить квантовое усреднение; для этого нужно ввести операторы

$$
\begin{aligned}
& \overline{\mathbf{G}_{1}} \stackrel{\text { def }}{=} \frac{1}{2 \pi} \int_{0}^{2 \pi} e^{-(i \tau / \hbar) \mathbf{G}_{0}} \mathbf{G}_{1} e^{(i \tau / \hbar) \mathbf{G}_{0}} d \tau, \\
& { }^{\#} \mathbf{G}_{1}=\frac{1}{2 \pi} \int_{0}^{2 \pi}(\pi-\tau) e^{-(i \tau / \hbar) \mathbf{G}_{0}} \mathbf{G}_{1} e^{(i \tau / \hbar) \mathbf{G}_{0}} d \tau .
\end{aligned}
$$

Тогда

$$
e^{(i \varepsilon / \hbar)^{\#} \mathbf{G}_{1}}\left(\mathbf{G}_{0}+\varepsilon \mathbf{G}_{1}\right) e^{-(i \varepsilon / \hbar)^{\#} \mathbf{G}_{1}}=\mathbf{G}_{0}+\varepsilon \overline{\mathbf{G}_{1}}+O\left(\varepsilon^{2}\right), \quad\left[\overline{\mathbf{G}_{1}}, \mathbf{G}_{0}\right]=0 .
$$

Таким образом, спектральная задача $(2.4)$ с точностью $O\left(\varepsilon^{3}\right)$ сводится к спектральной задаче для дважды усредненного возмушения $\overline{\mathbf{G}_{1}}$, а точнее, к задаче

$$
\left\{\begin{array}{l}
\mathbf{S}_{0} \varphi=\frac{\hbar(l+r) d}{2} \varphi, \\
\mathbf{G}_{0} \varphi=\hbar\left(m-\frac{l-r}{2}\right) \varphi \\
\overline{\mathbf{G}_{1} \varphi}=\zeta \varphi .
\end{array}\right.
$$

Здесь целые числа $m$ определяют спектр $\mathbf{G}_{0}$; они имеют вид

$$
m=r j_{2}-l j_{1},
$$

где $j_{1}, j_{2} \in \mathbb{Z}, 0 \leqslant j_{1} \leqslant r d-1,0 \leqslant j_{2} \leqslant l d-1$. Собственные значения $\zeta$ и собственные функции $\varphi$ оператора $\overline{\mathbf{G}_{1}}$ в $(2.11)$ связаны с исходными собственными значениями $\lambda$ и собственными функциями $\psi$ следуюшими формулами:

$$
\begin{aligned}
& \quad \begin{array}{l}
\lambda=\lambda_{n}+\varepsilon \cdot 2 \hbar^{2} d\left(m-\frac{l-r}{2}\right) \lambda_{n}^{2}+\varepsilon^{2} \cdot 2 \hbar d \lambda_{n}^{2}\left[\zeta+4 \hbar^{3} d\left(m-\frac{l-r}{2}\right)^{2} \lambda_{n}\right]+O\left(\varepsilon^{3}\right), \\
\quad \psi=\varphi+O(\varepsilon),
\end{array} \\
& \text { где } \lambda_{n}=\hbar(n+1+|k| / 2) .
\end{aligned}
$$


2.6. Дважды усредненное возмущение как элемент алгебры интегралов движения операторов “действие" $\mathbf{S}_{0}$ и $\mathbf{G}_{0}$. В результате двукратного применения квантового усреднения исходная система (2.4) сводится к квантовой интегрируемой системе с гамильтонианом $\overline{\mathbf{G}_{1}}$, который обладает двумя независимыми коммутирующими между собой квантовыми интегралами движения: $\mathbf{S}_{0}$ и $\mathbf{G}_{0}$.

Обозначим через $\mathcal{F}_{\text {quant }}$ алгебру всех операторов, коммутирующих с $\mathbf{S}_{0}$ и $~ с \mathbf{G}_{0}$.

ТЕОРема 2.1. Пусть $l, r$ - взаимно простые челье числа.

1. Алгебра $\mathcal{F}_{\text {quant }}$ операторов, коммутирующих с $\mathbf{S}_{0}$ и $c \mathbf{G}_{0}=l \mathbf{J}_{3}+r \mathbf{K}_{3}$, порождается следующими генераторами:

$$
\begin{aligned}
& \mathbf{A}_{1}=\mathbf{J}_{3}, \quad \mathbf{A}_{2}=\mathbf{K}_{3}, \quad \mathbf{A}_{3}=\mathbf{J}_{1}^{2}+\mathbf{J}_{2}^{2}, \quad \mathbf{A}_{4}=\mathbf{K}_{1}^{2}+\mathbf{K}_{2}^{2}, \\
& \mathbf{B}=\left(\mathbf{J}_{1}-i \mathbf{J}_{2}\right)^{r}\left(\mathbf{K}_{1}+i \mathbf{K}_{2}\right)^{l}, \quad \mathbf{C}=\left(\mathbf{J}_{1}+i \mathbf{J}_{2}\right)^{r}\left(\mathbf{K}_{1}-i \mathbf{K}_{2}\right)^{l} .
\end{aligned}
$$

Генераторы $\mathbf{A}$ әрмитовы: $\mathbf{A}=\mathbf{A}^{*}$, а $\mathbf{B}$ и $\mathbf{C}$ взаимно сопряжены: $\mathbf{B}=\mathbf{C}^{*}$.

2. Операторы $\mathbf{A}_{j}, \mathbf{B}, \mathbf{C}$ подчинены следующим полиномиальным перестановочныцм соотночениям:

$$
\begin{array}{ll}
{\left[\mathbf{A}_{i}, \mathbf{A}_{j}\right]=0,} & {[\mathbf{C}, \mathbf{B}]=f\left(\mathbf{A}_{1}, \mathbf{A}_{2}, \mathbf{A}_{3}, \mathbf{A}_{4}\right),} \\
\mathbf{A}_{1} \mathbf{B}=\mathbf{B}\left(\mathbf{A}_{1}-r \hbar\right), & \mathbf{C A}_{1}=\left(\mathbf{A}_{1}-r \hbar\right) \mathbf{C}, \\
\mathbf{A}_{2} \mathbf{B}=\mathbf{B}\left(\mathbf{A}_{2}+l \hbar\right), & \mathbf{C A}_{2}=\left(\mathbf{A}_{2}+l \hbar\right) \mathbf{C}, \\
\mathbf{A}_{3} \mathbf{B}=\mathbf{B}\left(\mathbf{A}_{3}+2 r \hbar \mathbf{A}_{1}-r^{2} \hbar^{2}\right), & \mathbf{C A}_{3}=\left(\mathbf{A}_{3}+2 r \hbar \mathbf{A}_{1}-r^{2} \hbar^{2}\right) \mathbf{C}, \\
\mathbf{A}_{4} \mathbf{B}=\mathbf{B}\left(\mathbf{A}_{4}-2 l \hbar \mathbf{A}_{2}-l^{2} \hbar^{2}\right), & \mathbf{C A}_{4}=\left(\mathbf{A}_{4}-2 l \hbar \mathbf{A}_{2}-l^{2} \hbar^{2}\right) \mathbf{C} .
\end{array}
$$

Здесь полином $f$ от четырех переменных определен формулой

$$
\begin{aligned}
f\left(A_{1}, A_{2}, A_{3}, A_{4}\right) & \stackrel{\text { def }}{=} \hbar\left(r \prod_{q=0}^{l-1}\left(A_{4}+(2 q+1) \hbar A_{2}-q(q+1) \hbar^{2}\right) \times\right. \\
& \times \sum_{j=0}^{r-1}\left(2 A_{1}+(r-1-2 j) \hbar\right) \prod_{p=j+1-r}^{j-1}\left(A_{3}+(2 p+1) \hbar A_{1}-p(p+1) \hbar^{2}\right)- \\
& -l \prod_{p=0}^{r-1}\left(A_{3}+(2 p+1) \hbar A_{1}-p(p+1) \hbar^{2}\right) \times \\
& \left.\times \sum_{j=0}^{l-1}\left(2 A_{2}+(l-1-2 j) \hbar\right) \prod_{q=j+1-l}^{j-1}\left(A_{4}+(2 q+1) \hbar A_{2}-q(q+1) \hbar^{2}\right)\right) .
\end{aligned}
$$

Соотношения (2.13) имеют четыре әлемента Казимира

$$
\mathbf{B C}-\rho\left(\mathbf{A}_{1}, \mathbf{A}_{2}, \mathbf{A}_{3}, \mathbf{A}_{4}\right), \quad l \mathbf{A}_{1}+r \mathbf{A}_{2}, \quad \mathbf{A}_{1}^{2}+\mathbf{A}_{3}, \quad \mathbf{A}_{2}^{2}+\mathbf{A}_{4},
$$


¿дe

$$
\begin{aligned}
\rho\left(A_{1}, A_{2}, A_{3}, A_{4}\right) \stackrel{\text { def }}{=} & \prod_{p=0}^{r-1}\left(A_{3}-(2 p+1) \hbar A_{1}-p(p+1) \hbar^{2}\right) \times \\
& \times \prod_{q=0}^{l-1}\left(A_{4}+(2 q+1) \hbar A_{2}-q(q+1) \hbar^{2}\right) .
\end{aligned}
$$

3. В представлении (2.12) әлементы Казимира выражаются через $\mathbf{S}_{0}, \mathbf{G}_{0}$ по формулам

$$
\begin{gathered}
\mathbf{B C}-\rho\left(\mathbf{A}_{1}, \mathbf{A}_{2}, \mathbf{A}_{3}, \mathbf{A}_{4}\right)=0, \quad l \mathbf{A}_{1}+r \mathbf{A}_{2}=\mathbf{G}_{0}, \\
\mathbf{A}_{1}^{2}+\mathbf{A}_{3}=\frac{1}{4}\left(\mathbf{S}_{0}-\mu\right)^{2}-\frac{\hbar^{2}}{4}, \quad \mathbf{A}_{2}^{2}+\mathbf{A}_{4}=\frac{1}{4}\left(\mathbf{S}_{0}+\mu\right)^{2}-\frac{\hbar^{2}}{4} .
\end{gathered}
$$

Если $k=0$, то $l=r=1$, и тогда $\mathbf{G}_{0}$ совпадает с третьей компонентой углового момента (см. формулы (2.7) в статье [2]): $\mathbf{G}_{0}=\mathbf{J}_{3}+\mathbf{K}_{3}=\mathbf{M}_{3}$. В этом случае алгебра $\mathcal{F}_{\text {quant }}$ совпадает с квадратичной алгеброй, описанной в работе [4].

Если $k \neq 0$, то хотя бы одно из чисел $l$ или $r$ больше 1 . Рассмотрим, например, простейший случай, когда $l=2, r=1$. Тогда полиномы $f(2.14)$ и $\rho(2.15)$ из теоремы $2.1-$ кубические:

$$
\begin{aligned}
& f\left(A_{1}, A_{2}, A_{3}, A_{4}\right)=2 \hbar\left(A_{1} A_{4}^{2}-4 A_{2} A_{3} A_{4}-2 \hbar^{2} A_{1} A_{4}+2 \hbar^{2} A_{2} A_{3}+3 \hbar^{2} A_{1} A_{2}^{2}\right), \\
& \rho\left(A_{1}, A_{2}, A_{3}, A_{4}\right)=\left(A_{3}-\hbar A_{1}\right)\left(A_{4}+\hbar A_{2}\right)\left(A_{4}+3 \hbar A_{2}-2 \hbar^{2}\right) .
\end{aligned}
$$

Вследствие теоремы 2.1 справедливо следующее

ПРЕДЛОЖЕНИЕ 2.2. 1. Для произвольного возмущающего потенциала $\mathcal{U}(q)$ дважды усредненное возмущение $\overline{\mathbf{G}_{1}}(2.10)$ является әлементом алгебры $\mathcal{F}_{\text {quant }}$, т.е. $\overline{\mathbf{G}_{1}}$ представим в виде функции от образующих $\mathbf{A}_{j}, \mathbf{B}, \mathbf{C}(2.12)$ этой алгебры.

2. В модельном примере $\mathcal{U}(q)=\gamma q_{1} q_{2}^{2}(2.2)$ оператор $\overline{\mathbf{G}_{1}}$ задается следующими формулами:

в случае $l=r=1$ (m.е. когда $k=0 u \mu=0)$

$$
\overline{\mathbf{G}_{1}}=\frac{\lambda^{2}}{2 \hbar d} \mathbf{S}_{0}\left(3 \mathbf{S}_{0}^{2}-6 \mathbf{A}_{1}^{2}-6 \mathbf{A}_{2}^{2}-4(\mathbf{B}+\mathbf{C})+\hbar^{2}\right) ;
$$

в cлучаe $l=2, r=1$

$$
\begin{aligned}
\overline{\mathbf{G}_{1}}= & \frac{\lambda^{2}}{2 \hbar d}\left(3 \mathbf{S}_{0}\left(\mathbf{S}_{0}^{2}-\mu^{2}\right)-6\left(\mathbf{S}_{0}+\mu\right) \mathbf{A}_{1}^{2}-6\left(\mathbf{S}_{0}-\mu\right) \mathbf{A}_{2}^{2}+\hbar^{2} \mathbf{S}_{0}\right)+ \\
& +\frac{\lambda^{3} \gamma}{2 \hbar d}\left(5 \mathbf{S}_{0}-\mu\right)(\mathbf{B}+\mathbf{C}) ;
\end{aligned}
$$


в случае $l=1, \quad r=2$

$$
\begin{aligned}
\overline{\mathbf{G}_{1}}=\frac{\lambda^{2}}{2 \hbar d}\left(3 \mathbf{S}_{0}\left(\mathbf{S}_{0}^{2}-\mu^{2}\right)-6\left(\mathbf{S}_{0}+\mu\right) \mathbf{A}_{1}^{2}-6\left(\mathbf{S}_{0}-\mu\right) \mathbf{A}_{2}^{2}+\hbar^{2} \mathbf{S}_{0}\right)- \\
-\frac{\lambda^{3} \gamma}{2 \hbar d}\left(5 \mathbf{S}_{0}+\mu\right)(\mathbf{B}+\mathbf{C}) ;
\end{aligned}
$$

в случае $l+r>3$

$$
\overline{\mathbf{G}_{1}}=\frac{\lambda^{2}}{2 \hbar d}\left(3 \mathbf{S}_{0}\left(\mathbf{S}_{0}^{2}-\mu^{2}\right)-6\left(\mathbf{S}_{0}+\mu\right) \mathbf{A}_{1}^{2}-6\left(\mathbf{S}_{0}-\mu\right) \mathbf{A}_{2}^{2}+\hbar^{2} \mathbf{S}_{0}\right) .
$$

Здесь $\mathbf{A}_{j}, \mathbf{B}, \mathbf{C}$ - образующие (2.12) алгебры $\mathcal{F}_{\text {quant }}$ (2.13).

Ниже в разделе 3 строятся неприводимые представления алгебры $\mathcal{F}_{\text {quant }}$ обыкновенными дифференциальными операторами в гильбертовых пространствах полиномов и соответствующие когерентные состояния. С их помощью можно вычислить собственные функции и собственные числа усредненного оператора $\overline{\mathbf{G}_{1}}$ в каждом из совместных собственных подпространств операторов $\mathbf{S}_{0}$ и $\mathbf{G}_{0}$ (на которых реализуются неприводимые представления алгебры $\left.\mathcal{F}_{\text {quant }}\right)$, а значит, и решить исходную спектральную задачу $(2.3)$.

\section{3. ПУАССОНОВА СТРУКТУРА И НЕПРИВОДИМЫЕ ПРЕДСТАВЛЕНИЯ АЛГЕБРЫ $\mathcal{F}$}

3.1. Алгебра $\mathcal{F}$ (классическая версия). В пределе при $\hbar \rightarrow 0$ соотношениям (2.1) отвечают следующие скобки Пуассона между координатами $q \in\left(\mathbb{R}^{3} \backslash 0\right), p \in \mathbb{R}^{3}$ :

$$
\left\{q_{j}, q_{k}\right\}=0, \quad\left\{p_{j}, q_{k}\right\}=\delta_{j k}, \quad\left\{p_{j}, p_{k}\right\}=-\mu \varepsilon_{j k l} \frac{q_{l}}{|q|^{3}} ;
$$

здесь $\mu \in \mathbb{R}$ - структурная константа. Соответствующий пуассонов тензор задает фазовое пространство $T^{*}\left(\mathbb{R}^{3} \backslash 0\right)$ с симплектической формой

$$
\sum_{j=1}^{3} d p_{j} \wedge d q_{j}+\frac{\mu}{|q|^{3}}\left(q_{1} d q_{2} \wedge d q_{3}+q_{2} d q_{3} \wedge d q_{1}+q_{3} d q_{1} \wedge d q_{2}\right) .
$$

На этом фазовом пространстве символ оператора “действие" $\mathbf{S}_{0}(2.5)$ - это функция $S_{0}(q, p)=\lambda|q||p|^{2}+\mu^{2} \lambda /|q|+|q| /(4 \lambda)$. Она находится в инволюции с компонентами вектор-функций

$$
M=(q \times p)-\mu \frac{q}{|q|}, \quad R=2 \lambda(q, p) p-\lambda q|p|^{2}+\frac{\mu^{2} \lambda q}{|q|^{2}}-\frac{2 \mu \lambda}{|q|}(q \times p)+\frac{1}{4 \lambda} q
$$

- символами операторов $\mathbf{M}$ и $\mathbf{R}$ (см. формулы (2.7) в работе [2]).

Вместо векторов $M$ и $R$ удобно ввести два новых вектора $J=(M+R) / 2$ и $K=(M-$ $R) / 2$ (это символы операторов $\mathbf{J}$ и $\mathbf{K}$; см. формулы (2.6) в работе [2]). Компоненты вектор-функций $J$ и $K$ порождают алгебру $s o(4)$ относительно скобки Пуассона:

$$
\left\{J_{j}, J_{k}\right\}=-\varepsilon_{j k l} J_{l}, \quad\left\{J_{j}, K_{k}\right\}=0, \quad\left\{K_{j}, K_{k}\right\}=-\varepsilon_{j k l} K_{l} .
$$

В этой алгебре имеются два элемента Казимира: $|J|^{2}$ и $|K|^{2}$. Они выражаются через функцию $S_{0}$ так: $|J|^{2}=\left(S_{0}-\mu\right)^{2} / 4,|K|^{2}=\left(S_{0}+\mu\right)^{2} / 4$. 
ТЕОРема 3.1. Пусть $l$ и $r$ - взаимно простые натуральные числа.

1. Алгебра $\mathcal{F}$ функиий на $T^{*}\left(\mathbb{R}^{3} \backslash 0\right)$, находящихся в инволющии с $S_{0}$ и $G_{0}=l J_{3}+$ $r K_{3}$, порождается следующими образующими:

$$
\begin{gathered}
A_{1}=J_{3}, \quad A_{2}=K_{3}, \quad A_{3}=J_{1}^{2}+J_{2}^{2}, \quad A_{4}=K_{1}^{2}+K_{2}^{2}, \\
B=\left(J_{1}-i J_{2}\right)^{r}\left(K_{1}+i K_{2}\right)^{l}, \quad C=\left(J_{1}+i J_{2}\right)^{r}\left(K_{1}-i K_{2}\right)^{l} .
\end{gathered}
$$

2. Образующие пуассоновой алгебры $\mathcal{F}$ подчинены полиномиальным соотношениям

$$
\begin{aligned}
\left\{A_{j}, A_{k}\right\} & =0 & & \{C, B\}=2 i\left(r^{2} A_{1} A_{3}^{r-1} A_{4}^{l}-l^{2} A_{2} A_{3}^{r} A_{4}^{l-1}\right), \\
\left\{A_{1}, B\right\} & =-i r B, & & \left\{A_{1}, C\right\}=i r C, \\
\left\{A_{2}, B\right\} & =i l B, & & \left\{A_{2}, C\right\}=-i l C, \\
\left\{A_{3}, B\right\} & =2 i r A_{1} B, & & \left\{A_{3}, C\right\}=-2 i r A_{1} C, \\
\left\{A_{4}, B\right\} & =-2 i l A_{2} B, & & \left\{A_{4}, C\right\}=2 i l A_{2} C .
\end{aligned}
$$

3. Соотношения (3.2) при любых (не обязательно иелочисленных) значениях $l \geqslant 1, \quad r \geqslant 1$ определяют скобки Пуассона на $\mathbb{R}^{6}$. Симплектические листы $\Omega \subset \mathbb{R}^{6}$ задаются четырьмя функииями Казимира

$$
\varkappa_{1}=b c-a_{3}^{r} a_{4}^{l}, \quad \varkappa_{2}=l a_{1}+r a_{2}, \quad \varkappa_{3}=a_{1}^{2}+a_{3}, \quad \varkappa_{4}=a_{2}^{2}+a_{4}
$$

$\left(\right.$ здесь $a_{1}, a_{2}, a_{3}, a_{4},(b+c) / 2,(b-c) /(2 i)$ - декартовы координаты на $\left.\mathbb{R}^{6}\right)$. Реализащия $a_{j}=A_{j}, \quad b=B, c=C$ (3.1) подчинена следующим условиям:

$$
\varkappa_{1}=0, \quad a_{3} \geqslant 0, \quad a_{4} \geqslant 0 .
$$

Рассмотрим область, где помимо условий (3.3) вьполнено дополнительное условие

$$
l^{2} \varkappa_{3}=r^{2} \varkappa_{4}
$$

В этой области симплектические листы параметризуются двумя константами $D$ и $\gamma$, причем $|\gamma| \leqslant \operatorname{lr} D ;$ замыкание листа задается уравнениями

$$
\bar{\Omega}=\left\{b c-a_{3}^{r} a_{4}^{l}=0, \quad l a_{1}+r a_{2}=\gamma, \quad a_{1}^{2}+a_{3}=\frac{r^{2} D^{2}}{4}, \quad a_{2}^{2}+a_{4}=\frac{l^{2} D^{2}}{4}\right\}
$$

причем в реализации (3.1) имеем

$$
\left.|J|\right|_{\Omega}=\left.\frac{S_{0}-\mu}{2}\right|_{\Omega}=\frac{r D}{2}, \quad|K|_{\Omega}=\left.\frac{S_{0}+\mu}{2}\right|_{\Omega}=\frac{l D}{2},\left.\quad\left(l J_{3}+r K_{3}\right)\right|_{\Omega}=\gamma .
$$

Если $|\gamma|=\operatorname{lr} D$, то листы $\Omega$ нульмерны и состоят из одной точки

$$
\left(a_{1}, a_{2}, a_{3}, a_{4}, b, c\right)=\left(\frac{\operatorname{sgn}(\gamma) r D}{2}, \frac{\operatorname{sgn}(\gamma) l D}{2}, 0,0,0,0\right)
$$


Если $0<|\gamma|<\operatorname{lrD}$, то при $l>1$ u $r>1$ листьл $\Omega$ диффеоморфнь иилиндру и получаются из замыкания (3.4) выкидыванием двух точек

$$
\begin{aligned}
& P \stackrel{\text { def }}{=}\left(a_{1}, a_{2}, a_{3}, a_{4}, b, c\right)=\left(\frac{\operatorname{sgn}(\gamma) r D}{2}, \frac{\gamma}{r}-\frac{\operatorname{sgn}(\gamma) l D}{2}, 0, \frac{|\gamma|(\operatorname{lr} D-|\gamma|)}{r^{2}}, 0,0\right), \\
& Q \stackrel{\text { def }}{=}\left(a_{1}, a_{2}, a_{3}, a_{4}, b, c\right)=\left(\frac{\gamma}{l}-\frac{\operatorname{sgn}(\gamma) r D}{2}, \frac{\operatorname{sgn}(\gamma) l D}{2}, \frac{|\gamma|(\operatorname{lr} D-|\gamma|)}{l^{2}}, 0,0,0\right) .
\end{aligned}
$$

Если $0<|\gamma|<\operatorname{lr} D$, то в случаях $l>1, r=1$ и $l=1, r>1$ листы диффеоморфны плоскости. В случае $l>1, \quad r=1$ листы получаются из замыкания (3.4) вькидыванием точки $Q$, а в случае $l=1, r>1$ листы получаются из $\bar{\Omega}$ выкидыванием точки $P$.

Если $0<|\gamma|<\operatorname{lrD}$, то при $l=r=1$ листы диффеоморфны сфере $\mathbb{S}^{2}$.

В случае $\gamma=0$ листы диффеоморфны чилиндру и получаются из $\bar{\Omega}$ (3.4) выкидьванием двух точек

$$
\left(a_{1}, a_{2}, a_{3}, a_{4}, b, c\right)=\left( \pm \frac{r D}{2}, \mp \frac{l D}{2}, 0,0,0,0\right)
$$

Во всех случаях выброшенные точки сами являются нульмерными симплектическими листами.

4. Кәлерова структура на листах $\Omega$ вводится с помощью комплексной координаты

$$
z=\frac{c}{\left(r D / 2+a_{1}\right)^{r}\left(l D / 2-a_{2}\right)^{l}} .
$$

Образующие $\left.a_{j}\right|_{\Omega},\left.b\right|_{\Omega},\left.c\right|_{\Omega}$ выражсаются через z следующим образом:

$$
\begin{aligned}
a_{1} & =\frac{r D}{2}-\frac{|\gamma|-\gamma}{2 l}-r a\left(|z|^{2}\right), \\
a_{2} & =-\frac{l D}{2}+\frac{|\gamma|+\gamma}{2 r}+l a\left(|z|^{2}\right), \\
a_{3} & =\left(\frac{|\gamma|-\gamma}{2 l}+r a\left(|z|^{2}\right)\right)\left(r D-\frac{|\gamma|-\gamma}{2 l}-r a\left(|z|^{2}\right)\right), \\
a_{4} & =\left(\frac{|\gamma|+\gamma}{2 r}+l a\left(|z|^{2}\right)\right)\left(l D-\frac{|\gamma|+\gamma}{2 r}-l a\left(|z|^{2}\right)\right), \\
b & =\left(r D-\frac{|\gamma|-\gamma}{2 l}-r a\left(|z|^{2}\right)\right)^{r}\left(l D-\frac{|\gamma|+\gamma}{2 r}-l a\left(|z|^{2}\right)\right)^{l} \bar{z} \\
c & =\left(r D-\frac{|\gamma|-\gamma}{2 l}-r a\left(|z|^{2}\right)\right)^{r}\left(l D-\frac{|\gamma|+\gamma}{2 r}-l a\left(|z|^{2}\right)\right)^{l} z
\end{aligned}
$$

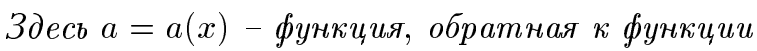

$$
x=x(a)=\frac{((|\gamma|-\gamma) /(2 l r)+a)^{r}((|\gamma|+\gamma) /(2 l r)+a)^{l}}{(D-(|\gamma|-\gamma) /(2 l r)-a)^{r}(D-(|\gamma|+\gamma) /(2 l r)-a)^{l}}
$$


на промежутке $0 \leqslant a \leqslant D-|\gamma| /($ lr $)$. Симплектическая структура на листах $\Omega$, соответствующая скобкам (3.2), имеет вид $\omega=i a^{\prime}\left(|z|^{2}\right) d \bar{z} \wedge d z$. Симплектическая площадь листа равна

$$
\frac{1}{2 \pi} \int_{\Omega} \omega=D-\frac{|\gamma|}{l r}
$$

3.2. Алгебра $\mathcal{F}_{\text {quant }}$ (квантовая версия). Согласно определению алгебры $\mathcal{F}_{\text {quant }}$ ее неприводимые представления реализуются на совместных собственных подпространствах операторов $\mathbf{S}_{0}$ и $\mathbf{G}_{0}$, где $\mathbf{S}_{0}$ принимает значения $\hbar(l+r) d / 2$, a $\mathbf{G}_{0}$ - значения $\hbar(m-(l-r) / 2) d$. Здесь $l, r-$ взаимно простые натуральные числа, $d \in \mathbb{N}$, а $m=$ $m\left(j_{1}, j_{2}\right)=r j_{2}-l j_{1}$, где $j_{1}, j_{2} \in \mathbb{Z}, 0 \leqslant j_{1} \leqslant r d-1,0 \leqslant j_{2} \leqslant l d-1$.

Отметим, что разным парам чисел $j_{1}, j_{2}$ может соответствовать одно и то же значение $m=m\left(j_{1}, j_{2}\right)$. Поэтому прежде всего введем обозначения для однозначной записи чисел $m$.

Лемма. Пусть $l, r-$ взаимно простые натуральные числа; $d \in \mathbb{N}$;

$$
m=r j_{2}-l j_{1}
$$

где $j_{1}, j_{2} \in \mathbb{Z}, \quad 0 \leqslant j_{1} \leqslant r d-1, \quad 0 \leqslant j_{2} \leqslant l d-1$.

Тогда существует единственная тройка чельх чисел $\left(m_{1}^{0}, m_{2}^{0}, \Delta m\right)$ такая, что

$$
m=r m_{2}^{0}-l m_{1}^{0}+\operatorname{lr} \Delta m, \quad 0 \leqslant m_{1}^{0} \leqslant r-1, \quad 0 \leqslant m_{2}^{0} \leqslant l-1, \quad|\Delta m| \leqslant d-1
$$

Верно и обратное: если некоторая тройка чельх чисел $\left(m_{1}^{0}, m_{2}^{0}, \Delta m\right)$ удовлетворяет условиям (3.6), то найдутся числа $j_{1}$ и $j_{2}$ такие, что выполнено условие (3.5), а именно

$$
j_{1}=m_{1}^{0}+r\left(\frac{|\Delta m|}{2}-\frac{\Delta m}{2}+j\right), \quad j_{2}=m_{2}^{0}+l\left(\frac{|\Delta m|}{2}+\frac{\Delta m}{2}+j\right)
$$

әде $j \in \mathbb{Z}, \quad 0 \leqslant j \leqslant d-1-|\Delta m|$.

3.2.1. Гипергеометрическое представление алгебры $\mathcal{F}_{\text {quant }}$. Пусть $l, r, d \in \mathbb{N}, m=$ $r m_{2}^{0}-l m_{1}^{0}+\operatorname{lr} \Delta m$, где $m_{1}^{0}, m_{2}^{0}, \Delta m \in \mathbb{Z}, 0 \leqslant m_{1}^{0} \leqslant r-1,0 \leqslant m_{2}^{0} \leqslant l-1,|\Delta m| \leqslant d-1$. Введем обозначения

$$
m_{1} \stackrel{\text { def }}{=} m_{1}^{0}+r\left(\frac{|\Delta m|}{2}-\frac{\Delta m}{2}\right), \quad m_{2} \stackrel{\text { def }}{=} m_{2}^{0}+l\left(\frac{|\Delta m|}{2}+\frac{\Delta m}{2}\right)
$$

и определим положительные числа

$$
\begin{aligned}
& \kappa_{0}=1 \\
& \kappa_{j}=(-1)^{(l+r) j} \frac{\left(1+m_{1}-r d\right)_{r j}\left(1+m_{2}-l d\right)_{l j}}{\left(1+m_{1}\right)_{r j}\left(1+m_{2}\right)_{l j}}, \quad 1 \leqslant j \leqslant d-1-|\Delta m| .
\end{aligned}
$$


Здесь операция $(\alpha)_{j}$ определяется формулой

$$
(\alpha)_{j} \equiv \alpha(\alpha+1) \ldots(\alpha+j-1), \quad(\alpha)_{0} \equiv 1 .
$$

Используя числа $\kappa_{j}$, введем гильбертово пространство $\mathcal{P}^{l, r}[d, m]$ полиномов степени не выше $d-1-|\Delta m|: f(\bar{z})=\sum_{j=0}^{d-1-|\Delta m|} f_{j} \bar{z}^{j}$. Здесь $z-$ стандартная комплексная координата на плоскости $z, \bar{z}$ обозначает комплексное сопряжение. Скалярное произведение в $\mathcal{P}^{l, r}[d, m]$ определим формулой

$$
\left\langle f, f^{\prime}\right\rangle_{\mathcal{P} l, r}[d, m] \stackrel{\text { def }}{=} \sum_{j=0}^{d-1-|\Delta m|} \frac{1}{\kappa_{j}} f_{j} \overline{f_{j}^{\prime}} .
$$

Tеорема 3.2. Пусть $l, r, d \in \mathbb{N} ; m=r m_{2}^{0}-l m_{1}^{0}+l r \Delta m$, әде $m_{1}^{0}, m_{2}^{0}, \Delta m \in \mathbb{Z}, 0 \leqslant$ $m_{1}^{0} \leqslant r-1, \quad 0 \leqslant m_{2}^{0} \leqslant l-1,|\Delta m| \leqslant d-1 ; m_{1}, m_{2}-$ числа $(3.7) ;$ и пусть $\hbar>0$.

Дифференциальные операторы

$$
\begin{aligned}
& \stackrel{\circ}{A}_{1}=\hbar\left(\frac{r d-1}{2}-m_{1}-r \bar{z} \frac{\partial}{\partial \bar{z}}\right), \\
& \stackrel{\circ}{A}_{2}=-\hbar\left(\frac{l d-1}{2}-m_{2}-l \bar{z} \frac{\partial}{\partial \bar{z}}\right), \\
& \stackrel{\circ}{A}_{3}=\frac{\hbar^{2}\left(r^{2} d^{2}-1\right)}{4}-\stackrel{\circ}{A}_{1}^{2}, \\
& \stackrel{\circ}{A}_{4}=\frac{\hbar^{2}\left(l^{2} d^{2}-1\right)}{4}-\stackrel{\circ}{A}_{2}^{2}, \\
& \stackrel{\circ}{B}=\hbar^{l+r} \prod_{p=0}^{r-1}\left(r d-m_{1}+p-r \bar{z} \frac{\partial}{\partial \bar{z}}\right) \prod_{q=0}^{l-1}\left(l d-m_{2}+q-l \bar{z} \frac{\partial}{\partial \bar{z}}\right) \bar{z}, \\
& \stackrel{\circ}{C}=\frac{\hbar^{l+r}}{\bar{z}} \prod_{p=0}^{r-1}\left(m_{1}-p+r \bar{z} \frac{\partial}{\partial \bar{z}}\right) \prod_{q=0}^{l-1}\left(m_{2}-q+l \bar{z} \frac{\partial}{\partial \bar{z}}\right)
\end{aligned}
$$

в пространстве $\mathcal{P}^{l, r}[d, m]$ задают неприводимое представление алгебры (2.13) со структурными константами $l$ и $r$, соответствующее симплектическому лисmy $\Omega(3.4)$ с параметрами $D=\hbar d, \gamma=\hbar(m-(l-r) d / 2)$. В этом представлении операторы $\stackrel{\circ}{A}_{j}$ әрмитовы: $\stackrel{\circ}{A}_{j}=\stackrel{\circ}{A}_{j}^{*}$, а $\stackrel{\circ}{B}$ и $\stackrel{\circ}{\mathrm{C}}$ взаимно сопряженши: $\stackrel{\circ}{B}=\stackrel{\circ}{C}^{*}$.

Это представление алгебры $\mathcal{F}_{\text {quant }}$ мы назовем гипергеометрическим.

Алгоритм построения представлений алгебры (2.13) в гильбертовых пространствах полиномов (в том числе и представления (3.11)) описывается в приложении Б статьи [2] и подробно изложен в работе [6].

3.2.2. Гипергеометрические когерентные состояния алгебры $\mathcal{F}_{\text {quant }}$. Предположим, что выполнены условия теоремы 3.2. Пусть задано произвольное представление соотношений (2.13) со структурными константами $l$ и $r$ в некотором гильбертовом пространстве $\mathbb{L}_{-}^{l, r}$. Генераторы этого представления обозначим через $\mathbb{A}_{j}, \mathbb{B}, \mathbb{C}$. Предположим, что в этом представлении операторы $\mathbb{A}_{j}$ эрмитовы, а операторы $\mathbb{B}$ и $\mathbb{C}$ взаимно сопряжены. 
Пусть $\chi_{0} \in \mathbb{L}_{-}^{l, r}-$ нормированный “вакуумный” вектор, подчиненный уравнениям

$$
\begin{aligned}
\mathbb{C}_{\chi_{0}} & =0 \\
\mathbb{A}_{1} \chi_{0} & =\hbar\left(\frac{r d-1}{2}-m_{1}\right) \chi_{0}, \quad \mathbb{A}_{3} \chi_{0}=\hbar^{2}\left(\frac{r^{2} d^{2}-1}{4}-\left(\frac{r d-1}{2}-m_{1}\right)^{2}\right) \chi_{0}, \\
\mathbb{A}_{2} \chi_{0} & =-\hbar\left(\frac{l d-1}{2}-m_{2}\right) \chi_{0}, \quad \mathbb{A}_{4} \chi_{0}=\hbar^{2}\left(\frac{l^{2} d^{2}-1}{4}-\left(\frac{l d-1}{2}-m_{2}\right)^{2}\right) \chi_{0} .
\end{aligned}
$$

Положим

$$
\begin{aligned}
\mathfrak{H}_{z} & \stackrel{\text { def }}{=} \sum_{j=0}^{d-1-|\Delta m|} \frac{1}{\left(1+m_{1}\right)_{r j}\left(1+m_{2}\right)_{l j}}\left(\frac{z \mathbb{B}}{\hbar^{l+r}}\right)^{j} \chi_{0}= \\
& ={ }_{0} F_{l+r-1}\left(\left(\frac{1+m_{1}}{r}, \ldots, \frac{r+m_{1}}{r}, \frac{1+m_{2}}{l}, \ldots, \frac{l+m_{2}}{l}\right)^{\prime} ; \frac{z \mathbb{B}}{l^{l} r^{r} \hbar^{l+r}}\right) \chi_{0} .
\end{aligned}
$$

Здесь ${ }_{s} F_{t}\left(\alpha_{1}, \ldots, \alpha_{s} ; \beta_{1}, \ldots, \beta_{t} ; y\right)$ - гипергеометрический ряд [7]:

$$
{ }_{s} F_{t}\left(\alpha_{1}, \ldots, \alpha_{s} ; \beta_{1}, \ldots, \beta_{t} ; y\right) \stackrel{\text { def }}{=} \sum_{j \geqslant 0}^{\infty} \frac{\left(\alpha_{1}\right)_{j} \ldots\left(\alpha_{s}\right)_{j}}{j !\left(\beta_{1}\right)_{j} \ldots\left(\beta_{t}\right)_{j}} y^{j}
$$

штрих в выражении ${ }_{s} F_{t-1}\left(\alpha_{1}, \ldots, \alpha_{s} ;\left(\beta_{1}, \ldots, \beta_{t}\right)^{\prime} ; y\right)$ обозначает, что по меньшей мере один из параметров $\beta_{1}, \ldots, \beta_{t}$ гипергеометрического ряда равен единице и один из таких единичных параметров опускается; операция $(\alpha)_{j}$ определена формулой (3.9). Мы называем семейство векторов $\mathfrak{H}_{z}(3.12)$ гипергеометрическими когерентными состояниями.

Вычисление показывает, что

$$
\left\langle\mathbb{B}^{j} \chi_{0}, \mathbb{B}^{i} \chi_{0}\right\rangle_{\mathbb{L}_{-}^{l, r}}=\delta_{j i} \hbar^{2(l+r)} \kappa_{j}\left[\left(1+m_{1}\right)_{r j}\left(1+m_{2}\right)_{l j}\right]^{2}
$$

Введем ортонормированные векторы

$$
\chi_{j}=(-1)^{l j} \frac{\mathbb{B}^{j} \chi_{0}}{\left\|\mathbb{B}^{j} \chi_{0}\right\|_{\mathbb{L}_{-}^{l, r}}}, \quad 0 \leqslant j \leqslant d-1-|\Delta m| .
$$

Их линейная оболочка $\mathbb{L}_{-}^{l, r}[d, m]$ образует подпространство в $\mathbb{L}_{-}^{l, r}$, на котором реализуется неприводимое представление алгебры $\mathcal{F}_{\text {quant }}$.

Гипергеометрические когерентные состояния $\mathfrak{H}_{z}(3.12)$ могут быть разложены по базису (3.14):

$$
\mathfrak{H}_{z}=\sum_{j=0}^{d-1-|\Delta m|} \overline{g_{j}(\bar{z})} \chi_{j}
$$


Здесь $\left\{g_{j}\right\}$ - ортонормированный базис в $\mathcal{P}^{l, r}[d, m]$, состоящий из мономов

$$
g_{j}(\bar{z})=(-1)^{l j} \sqrt{\kappa_{j}} \bar{z}^{j}
$$

В частности, если представление $\mathbb{A}_{j}=\mathbf{A}_{j}, \mathbb{B}=\mathbf{B}, \mathbb{C}=\mathbf{C}(2.12)$ реализовано в гильбертовом пространстве $\mathcal{L}$ - и числа $k, n$ связаны с числами $l, r, d$ формулами $(2.9)$, т.е. $n+1+|k|=(l+r) d / 2, k=(l-r) / 2$, то векторы $\chi_{j}(3.14)$ имеют вид

$$
\chi_{j} \stackrel{\text { def }}{=} \psi_{m_{1}+r j, m_{2}+l j}^{n},
$$

где $\psi_{j_{1}, j_{2}}^{n}$ - функции (2.11) из работы [2], а $m_{1}, m_{2}$ - числа (3.7). В этом случае векторы $\chi_{j}$ образуют ортонормированный базис в подпространстве $\mathcal{L}_{-}^{l, r}[d, m] \subset \mathcal{L}_{-}^{k}$, где реализуется неприводимая компонента представления (2.12). Это совместное собственное подпространство операторов $\mathbf{S}_{0}$ и $\mathbf{G}_{0}$ :

$$
\mathcal{L}_{-}^{l, r}[d, m]=\left\{\chi \in \mathcal{L}_{-} \mid \mathbf{S}_{0} \chi=\hbar\left(n+1+\frac{|k|}{2}\right) \chi, \quad \mathbf{G}_{0} \chi=\hbar\left(m-\frac{k}{2}\right) \chi\right\} .
$$

Теперь заметим, что скалярное произведение двух когерентных состояний (3.12) задается формулой

$$
\left\langle\mathfrak{H}_{z^{\prime}}, \mathfrak{H}_{z^{\prime \prime}}\right\rangle_{\mathbb{L}_{-}}=\mathfrak{K}\left(z^{\prime} \overline{z^{\prime \prime}}\right), \quad \mathfrak{K}(x) \stackrel{\text { def }}{=} \sum_{j=0}^{d-1-|\Delta m|} \kappa_{j} x^{j} .
$$

Полином $\mathfrak{K}(x)$ является единственным решением гипергеометрического уравнения

$$
\begin{gathered}
x \prod_{p=0}^{r-1}\left(r d-r-m_{1}+p-r x \frac{d}{d x}\right) \prod_{q=0}^{l-1}\left(l d-l-m_{2}+q-l x \frac{d}{d x}\right) \mathfrak{K}- \\
-\prod_{p=0}^{r-1}\left(m_{1}-p+r x \frac{d}{d x}\right) \prod_{q=0}^{l-1}\left(m_{2}-q+l x \frac{d}{d x}\right) \mathfrak{K}=0
\end{gathered}
$$

удовлетворяюшим условию нормировки $\mathfrak{K}(0)=1$. Отсюда следует, что

$$
\begin{gathered}
\mathfrak{K}(x)={ }_{l+r} F_{l+r-1}\left(\frac{1+m_{1}}{r}-d, \ldots, \frac{r+m_{1}}{r}-d, \frac{1+m_{2}}{l}-d, \ldots, \frac{l+m_{2}}{l}-d ;\right. \\
\left.\left(\frac{1+m_{1}}{r}, \ldots, \frac{r+m_{1}}{r}, \frac{1+m_{2}}{l}, \ldots, \frac{l+m_{2}}{l}\right)^{\prime} ;(-1)^{l+r} x\right),
\end{gathered}
$$

где ${ }_{s} F_{t}$-гипергеометрическая функция (3.13), а штрих обозначает ту же операцию, что и в формуле (3.12). 
ПРЕДЛОЖЕНИЕ 3.1. Полиномы $\mathfrak{K}(x)$ обладают воспроизводящим свойством:

$$
\left\langle\mathfrak{K}\left(z^{\prime} \bar{z}\right), \mathfrak{K}\left(z^{\prime \prime} \bar{z}\right)\right\rangle_{\mathcal{P} l, r[d, m]}=\mathfrak{K}\left(z^{\prime} \overline{z^{\prime \prime}}\right) .
$$

Здесь $\mathfrak{K}\left(z^{\prime} \bar{z}\right)$ и $\mathfrak{K}\left(z^{\prime \prime} \bar{z}\right)$ рассматриваются как әлементы гильбертова пространства $\mathcal{P}^{l, r}[d, m]$ полиномов от $\bar{z}$ со скалярным произведением (3.10).

Теперь мы укажем явный вид преобразования, сплетаюшего представления алгебры $\mathcal{F}_{\text {quant }}$ c неприводимыми представлениями. Эта конструкция основана на следуюшем свойстве когерентных состояний $\mathfrak{H}_{z}$ :

$$
\mathbb{A}_{j} \mathfrak{H}_{z}=\bar{\circ}_{A_{j}} \mathfrak{H}_{z}, \quad j=1,2,3,4 ; \quad \mathbb{B H}_{z}=\stackrel{\bar{C}}{C} \mathfrak{H}_{z} ; \quad \mathbb{C H}_{z}=\overline{\bar{B}} \mathfrak{H}_{z}
$$

В этих формулах операторы $\bar{\circ} \underset{A_{j}}{ }, \stackrel{\bar{\circ}}{B}, \stackrel{\bar{\circ}}{C}$ действуют по переменной $z$ и получаются комплексным сопряжением из операторов $\stackrel{\circ}{A}_{j}, \stackrel{\circ}{B}, \stackrel{\circ}{C}$, заданных формулами (3.11).

Обозначим через $\mathfrak{h}$ мономорфизм $\mathfrak{h}: \mathcal{P}^{l, r}[d, m] \rightarrow \mathbb{L}_{-}^{l, r}$, который переводит каждый базисньй моном $g_{j}(3.15)$ в вектор $\chi_{j}(3.14)$, т.е.

$$
\mathfrak{h}\left(g_{j}\right)=\chi_{j}, \quad j=0,1, \ldots, d-1-|\Delta m| .
$$

Теорема 3.3. 1. Когерентное преобразование $\mathfrak{h}: \mathcal{P}^{l, r}[d, m] \rightarrow \mathbb{L}_{-}^{l, r}[d, m]$, заданное формулой (3.17), унитарно. Обратное преобразование задается формулой

$$
\left(\mathfrak{h}^{-1} \chi\right)(\bar{z})=\left\langle\chi, \mathfrak{H}_{z}\right\rangle_{\mathbb{L}_{-}}
$$

для любого $\chi \in \mathbb{L}_{-}^{l, r}[d, m]$.

Для любого фиксированного $w \in \mathbb{C}$ преобразование $\mathfrak{h}$ переводит гипергеометрический полином $\mathfrak{K}(w \bar{z})$ в гипергеометрическое когерентное состояние $\mathfrak{H}_{w}$.

2. Выполнены формульи коммутации

$$
\mathbb{A}_{j} \circ \mathfrak{h}=\mathfrak{h} \circ \stackrel{\circ}{A}_{j}, \quad j=1,2,3,4 ; \quad \mathbb{B} \circ \mathfrak{h}=\mathfrak{h} \circ \stackrel{\circ}{B} ; \quad \mathbb{C} \circ \mathfrak{h}=\mathfrak{h} \circ \stackrel{\circ}{C},
$$

т.е. $\mathfrak{h}$ сплетает абстрактное представление $\mathbb{A}_{j}, \mathbb{B}, \mathbb{C}$ алгебрь (2.13) в гильбертовом пространстве $\mathbb{L}_{-}^{l, r}$ с гипергеометрическим (неприводимым) представлением

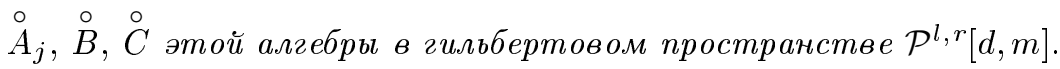

В приложении Б работы [2] (подробнее см. [6]) приведен алгоритм построения когерентных состояний (3.12). Ниже в приложении к данной статье описана реализация операторов $(2.12)$ в пространстве $L^{2}\left(\mathbb{R}^{4}\right)$ и выписаны гипергеометрические когерентные состояния (3.12) в этой реализации. 


\section{4. РЕШЕНИЕ СПЕКТРАЛЬНОЙ ЗАДАЧИ С ПОМОЩЬЮ КОГЕРЕНТНОГО ПРЕОБРАЗОВАНИЯ}

4.1. Когерентное преобразование дважды усредненной задачи. В силу утверждения 1 теоремы 3.3 спектральная задача в любом представлении алгебры (2.13) может быть переформулирована в терминах дифференциального уравнения в пространстве полиномов $\mathcal{P}^{l, r}[d, m]$.

Объясним это подробнее. Пусть исходная спектральная задача имеет вид

$$
f(\stackrel{3}{\mathbb{B}}, \stackrel{1}{\mathbb{A}}, \stackrel{1}{\mathbb{C}}) \varphi=\zeta \varphi, \quad \varphi \in \mathbb{L}_{-}^{l, r}[d, m]
$$

где $\mathbb{A}=\left(\mathbb{A}_{1}, \mathbb{A}_{2}, \mathbb{A}_{3}, \mathbb{A}_{4}\right), \mathbb{B}, \mathbb{C}$ - некоторое представление соотношений $(2.13)$ в гильбертовом пространстве $\mathbb{L}_{-}^{l, r}$, а $f$ - функция шести переменных, например, полином. Тогда когерентное преобразование (3.17)

$$
\varphi=\mathfrak{h}(\Phi)
$$

сводит задачу (4.1) к следующей задаче в пространстве полиномов:

$$
\begin{aligned}
& \begin{array}{lll}
3 & 2 & 1
\end{array} \\
& f(\stackrel{B}{B}, \stackrel{\leftrightarrow}{C}, \stackrel{\circ}{C}) \Phi=\zeta \Phi, \quad \Phi \in \mathcal{P}^{l, r}[d, m] .
\end{aligned}
$$

Здесь $\stackrel{\circ}{A}, \stackrel{\circ}{B}, \stackrel{\circ}{C}$ - неприводимое представление соотношений (2.13), заданное формулами (3.11).

Решение задачи (4.3) - некоторый полином, и его можно разложить по базису мономов $g_{j}(3.15)$ :

$$
\Phi(\bar{z})=\sum_{j=0}^{d-1-|\Delta m|} \Phi^{j} g_{j}(\bar{z})
$$

Тогда формула (4.2) дает для решения задачи (4.1) выражение

$$
\varphi=\sum_{j=0}^{d-1-|\Delta m|} \Phi^{j} \chi_{j}
$$

где $\left\{\chi_{j}\right\}$ - ортонормированный базис $(3.14)$ в $\mathbb{L}_{-}^{l, r}[d, m]$.

Рассмотрим теперь нашу конкретную спектральную задачу (2.11). В этом случае мы используем представление $\mathbb{A}_{j}=\mathbf{A}_{j}, \mathbb{B}=\mathbf{B}, \mathbb{C}=\mathbf{C}(2.12)$ алгебры $\mathcal{F}_{\text {quant }}$, заданное в пространстве $\mathbb{L}_{-}^{l, r}=\mathcal{L}_{-}^{l, r}$. Уравнение $\overline{\mathbf{G}_{1}} \varphi=\zeta \varphi$ представляется в виде (4.1). Для наглядности остановимся на модельном примере $(2.2)$, в котором усредненный гамиль-

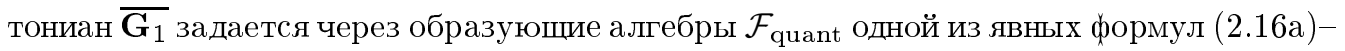
$(2.16 г)$. Заметим, что любые векторы вида $\varphi=\mathfrak{h}(\Phi)$ автоматически дают точные решения первых двух уравнений (2.11) при любых $\Phi$. Третье уравнение (2.11) принимает вид (4.3), т.е. 
в случае $l=r=1$

$$
\begin{aligned}
\bar{z}\left(\bar{z}^{2}+\right. & 3 \bar{z}+1) \frac{d^{2} \Phi}{d \bar{z}^{2}}+\left((|m|-2 d+3) \bar{z}^{2}+3(|m|-d+2) \bar{z}+|m|+1\right) \frac{d \Phi}{d \bar{z}}+ \\
& +\left((d-1)(d-1-|m|) \bar{z}-\frac{3}{2}(d-|m|)(|m|+1)+\frac{1}{2}+\frac{\zeta}{2 \hbar^{4} d^{2}}\right) \Phi=0
\end{aligned}
$$

в случае $l=2, r=1$

$$
\begin{aligned}
28 \hbar^{2} \gamma & d \bar{z}^{2}\left(\bar{z}^{2}-1\right) \frac{d^{3} \Phi}{d \bar{z}^{3}}- \\
& -\bar{z}\left(14 \hbar^{2} \gamma d\left(6 d-2 m_{1}-2 m_{2}-11\right) \bar{z}^{2}-24 \bar{z}+14 \hbar^{2} \gamma d\left(2 m_{1}+2 m_{2}+5\right)\right) \frac{d^{2} \Phi}{d \bar{z}^{2}}+ \\
& +\left(7 \hbar^{2} \gamma d\left(12 d^{2}+m_{2}^{2}+4 m_{1} m_{2}-8 d m_{1}-8 d m_{2}+10 m_{1}+11 m_{2}-32 d+22\right) \bar{z}^{2}+\right. \\
& \left.+8\left(2 m_{1}+2 m_{2}-3 d+5\right) \bar{z}-7 \hbar^{2} \gamma d\left(m_{2}^{2}+3 m_{1} m_{2}+2 m_{1}+4 m_{2}+2\right)\right) \frac{d \Phi}{d \bar{z}}- \\
& -\left(7 \hbar^{2} \gamma d\left(d-1-m_{1}\right)\left(2 d-2-m_{2}\right)\left(2 d-1-m_{2}\right) \bar{z}-\right. \\
& \left.-2\left(d-1-2 m_{1}\right)^{2}-\left(2 d-1-2 m_{2}\right)^{2}+6 d^{2}+1-\frac{16 \zeta}{27 \hbar^{4} d^{2}}\right) \Phi(\bar{z})=0 ;
\end{aligned}
$$

в случае $l=1, r=2$

$$
\begin{aligned}
28 \hbar^{2} \gamma & d \bar{z}^{2}\left(1-\bar{z}^{2}\right) \frac{d^{3} \Phi}{d \bar{z}^{3}}+ \\
& +\bar{z}\left(14 \hbar^{2} \gamma d\left(6 d-2 m_{1}-2 m_{2}-11\right) \bar{z}^{2}+24 \bar{z}+14 \hbar^{2} \gamma d\left(2 m_{1}+2 m_{2}+5\right)\right) \frac{d^{2} \Phi}{d \bar{z}^{2}}- \\
& -\left(7 \hbar^{2} \gamma d\left(12 d^{2}+m_{1}^{2}+4 m_{1} m_{2}-8 d m_{1}-8 d m_{2}+11 m_{1}+10 m_{2}-32 d+22\right) \bar{z}^{2}-\right. \\
& \left.-8\left(2 m_{1}+2 m_{2}-3 d+5\right) \bar{z}-7 \hbar^{2} \gamma d\left(m_{1}^{2}+3 m_{1} m_{2}+4 m_{1}+2 m_{2}+2\right)\right) \frac{d \Phi}{d \bar{z}}+ \\
& +\left(7 \hbar^{2} \gamma d\left(d-1-m_{2}\right)\left(2 d-2-m_{1}\right)\left(2 d-1-m_{1}\right) \bar{z}+\right. \\
& \left.+2\left(d-1-2 m_{2}\right)^{2}+\left(2 d-1-2 m_{1}\right)^{2}-6 d^{2}-1+\frac{16 \zeta}{27 \hbar^{4} d^{2}}\right) \Phi(\bar{z})=0 ;
\end{aligned}
$$

в случае $l+r>3$

$$
\begin{aligned}
& \left((l+r)\left(3 l r d^{2}+1\right)-12 l\left(\frac{1-r d}{2}+m_{1}+r \bar{z} \frac{d}{d \bar{z}}\right)^{2}-\right. \\
& \left.-12 r\left(\frac{1-l d}{2}+m_{2}+l \bar{z} \frac{d}{d \bar{z}}\right)^{2}-\frac{16 \zeta}{\hbar^{4} d^{2}(l+r)^{2}}\right) \Phi(\bar{z})=0 .
\end{aligned}
$$

Отметим, что в случае $l=r=1$ дифференциальное уравнение (4.6а) - известное уравнение Хейна ${ }^{1)}$.

\footnotetext{
1) Точнее, оригинальное уравнение Хейна [8] получается из (4.6а) после линейной замены переменной $\bar{z} \rightarrow-(3+\sqrt{5}) \bar{z} / 2$.
} 
4.2. Асимптотика спектра и собственных функций. В любом из перечисленных четырех случаев всего имеется $d-|\Delta m|$ собственных чисел и независимых полиномиальных решений дифференциального уравнения (4.6а)-(4.6г). Обозначим эти числа через $\zeta_{j}$, а полиномы - через $\Phi_{j}, j=0,1, \ldots, d-1-|\Delta m|$.

Разложим $\Phi_{j}$ согласно (4.4):

$$
\Phi_{j}(\bar{z})=\sum_{i=0}^{d-1-|\Delta m|} \Phi_{j}^{i} g_{i}(\bar{z}) .
$$

Тогда собственные функции $\varphi_{j} \in \mathcal{L}_{-}^{l, r}[d, m]$ задачи (2.11) получим по формуле (4.5), т.е. $\varphi_{j}=\sum_{p=0}^{d-1-|\Delta m|} \Phi_{j}^{p} \chi_{p}$, где $\left\{\chi_{j}\right\}-$ базис (3.16) в подпространстве $\mathcal{L}_{-}^{l, r}[d, m]$.

В частном случае $l+r>3$ нормированные решения уравнения (4.6г) - мономы $(3.15)$ : $\Phi_{j}(\bar{z})=g_{j}(\bar{z}) ;$ поэтому $\Phi_{j}^{p}=\delta_{j}^{p}$. Следовательно, нормированные собственные функции задачи (2.11) - функции (3.16): $\varphi_{j}=\chi_{j}$. Соответствуюшие собственные значения равны

$$
\begin{aligned}
\zeta_{j}= & \frac{\hbar^{4} d^{2}(l+r)^{2}}{16}\left((l+r)\left(3 l r d^{2}+1\right)-12 l\left(\frac{1-r d}{2}+m_{1}+r j\right)^{2}-\right. \\
& \left.-12 r\left(\frac{1-l d}{2}+m_{2}+l j\right)^{2}\right) .
\end{aligned}
$$

Tеорема 4.1. Пусть $\varepsilon \ll 1, ~ \hbar>0, \quad \mu=\hbar k / 2$, где $k \in \mathbb{Z}$. Тогда решение спектральной задачи (2.3) для квантовой частицы в поле Кулона-Дирака, возмущенном однородным магнитным полем и кубическим потенииалом (2.2), задается формулами

$$
\begin{aligned}
& \mathcal{E}=\mathcal{E}_{j, n, m}=-\frac{1}{4 \lambda_{n}^{2}}+\varepsilon \hbar^{2} \frac{m d-k / 2}{\lambda_{n}}+\varepsilon^{2}\left(\hbar^{4}\left(m d-\frac{k}{2}\right)^{2}+\frac{\zeta_{j} d \hbar}{\lambda_{n}}\right)+O\left(\varepsilon^{3}\right), \\
& \psi=\psi_{j, n, m}=\sum_{p=0}^{d-1-|\Delta m|} \Phi_{j}^{i} \chi_{i}+O(\varepsilon), \quad n \in \mathbb{Z}_{+}, \quad 0 \leqslant j \leqslant d-1-|\Delta m| .
\end{aligned}
$$

Здесь $\lambda_{n}=\hbar(n+1+|k| / 2)$, а $\zeta_{j} u \Phi_{j}-j$-е собственное число и полиномиальное решение одного из уравнений (4.6а)-(4.6г) (в зависимости от значений чисел $l, r$ ). Взаимно простые натуральные числа $l$ и $r$ и натуральное число $d$ определяются для каждого значения $n$ формулами (2.8), (2.9) (в случае $k=0$ следует положить $l=r=1, \quad d=n+1) ;$ число $m$ - любое челое, представимое в виде (3.6); $m_{1}, m_{2}$ - числа (3.7); $\chi_{j}$ - функции (3.16); $\Phi_{j}^{i}$ - коэффициенты разложсения (4.7); остаток $O\left(\varepsilon^{3}\right)$ оченивается в норме $\mathcal{L}_{-}$.

В частности, результат этой теоремы применим и к “чистому" эффекту Зеемана, когда кубический потенциал в $(2.3)$ отсутствует $(\gamma=0)$. Здесь в случае $l=r=1$ числа $\zeta_{j}-$ собственные значения уранения Хейна (4.6a), а $\Phi_{j}$ - соответствуюшие полиномы Хейна. В остальных случаях (когда $l+r \geqslant 3$ ) числа $\zeta_{j}$ задаются следуюшими формулами:

6 Теоретическая и математическая физика, т. 142, № 1, 2005 г. 
в случае $l=2, r=1$

$$
\begin{aligned}
\zeta_{j}= & -\frac{27 \hbar^{4} d^{2}}{16}\left[24 j^{2}+8\left(2 m_{1}+2 m_{2}-3 d+2\right) j+2\left(d-1-2 m_{1}\right)^{2}+\right. \\
& \left.+\left(2 d-1-2 m_{2}\right)^{2}-6 d^{2}-1\right]
\end{aligned}
$$

в случае $l=1, r=2$

$$
\begin{aligned}
\zeta_{j}= & -\frac{27 \hbar^{4} d^{2}}{16}\left[24 j^{2}+8\left(2 m_{1}+2 m_{2}-3 d+2\right) j+2\left(d-1-2 m_{2}\right)^{2}+\right. \\
& \left.+\left(2 d-1-2 m_{1}\right)^{2}-6 d^{2}-1\right]
\end{aligned}
$$

в случае $l+r>3$

$$
\begin{aligned}
\zeta_{j}= & \frac{(l+r)^{2} \hbar^{4} d^{2}}{16}\left[(l+r)\left(3 l r d^{2}+1\right)-12 l\left(\frac{1-r d}{2}+m_{1}+r j\right)^{2}-\right. \\
& \left.-12 r\left(\frac{1-l d}{2}+m_{2}+l j\right)^{2}\right] .
\end{aligned}
$$

При этом коэффициенты $\Phi_{j}^{i}$ во всех случаях $l+r \geqslant 3$ имеют вид $\Phi_{j}^{i}=\delta_{j}^{i}$.

Таким образом, мы развили теорию эффекта Зеемана для частицы в поле электромагнитного монополя. Показано, что вырождение старшей части спектра может быть снято только в далеких (квадратичных или выше) членах теории возмущений по магнитному полю. Снятие вырождения контролируется динамической алгеброй с полиномиальными перестановочными соотношениями (2.13), структура которой определяется арифметической пропорцией (2.8) между главным квантовым числом $n$ и квантовым числом $k$ магнитного заряда. Неприводимые представления (3.11) динамической алгебры реализуются в пространстве полиномов и задают модельные дифференциальные уравнения (4.6) с полиномиальными решениями, которые определяют главный член асимптотики собственных функций в эффекте Зеемана. Дополнительная асимптотика построенных решений (4.8) при больших квантовых числах $n, k \sim 1 / \hbar$ может быть получена с помощью гипергеометрических когерентных состояний (П.1) (см. приложение) по той же схеме, которая применялась в работе [4] для случая нулевого магнитного заряда, т.е. когда $k=0$.

\section{ПРИЛОЖЕНИЕ}

\section{Представление алгебры $\mathcal{F}_{\text {quant }}$ и когерентные состояния в $L^{2}\left(\mathbb{R}^{4}\right)$}

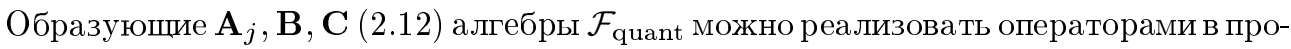
странстве $L^{2}\left(\mathbb{R}^{4}\right)$. В этом случае $\mathbf{A}_{j}, \mathbf{B}, \mathbf{C}$ - дифференциальные операторы, действующие по переменным $u=\left(u_{1}, u_{2}, u_{3}, u_{4}\right) \in \mathbb{R}^{4}$ :

$$
\begin{gathered}
\mathbf{A}_{1}=\widehat{A_{1}}=\widehat{J_{3}}, \quad \mathbf{A}_{2}=\widehat{A_{2}}=\widehat{K_{3}}, \quad \mathbf{A}_{3}={\widehat{A_{3}}}^{=}{\widehat{J_{1}}}^{2}+{\widehat{J_{2}}}^{2}, \quad \mathbf{A}_{4}={\widehat{A_{4}}}^{2}{\widehat{K_{1}}}^{2}+{\widehat{K_{2}}}^{2}, \\
\mathbf{B}=\widehat{B}=\left(\widehat{J_{1}}-i \widehat{J_{2}}\right)^{r}\left(\widehat{K_{1}}+i \widehat{K_{2}}\right)^{l}, \quad \mathbf{C}=\widehat{C}=\left(\widehat{J_{1}}+i{\widehat{J_{2}}}^{r}\left({\widehat{K_{1}}}^{-i \widehat{K}_{2}}\right)^{l},\right.
\end{gathered}
$$


где

$$
\hat{J}=\frac{1}{2}(\widehat{M}+\widehat{R}), \quad \widehat{K}=\frac{1}{2}(\widehat{M}-\widehat{R}),
$$

а $\widehat{M}, \widehat{R}$ задаются формулами

$$
\widehat{M}=\frac{1}{2} C(u) \widehat{w}, \quad \widehat{R}=\frac{1}{4 \omega}\left({ }^{\sigma} \widehat{w}+\omega^{2} \sigma_{u}\right) .
$$

Здесь $\omega=1 / \lambda$, матрица $C(u)$ и вектор ${ }^{\sigma} u$ для каждого $u \in \mathbb{R}^{4}$ определяются как

$$
C(u)=\left(\begin{array}{cccc}
u_{4} & -u_{3} & u_{2} & -u_{1} \\
-u_{3} & -u_{4} & u_{1} & u_{2} \\
u_{2} & -u_{1} & -u_{4} & u_{3}
\end{array}\right), \quad \sigma_{u}=\left(\begin{array}{c}
2\left(u_{1} u_{3}+u_{2} u_{4}\right) \\
2\left(u_{1} u_{4}-u_{2} u_{3}\right) \\
u_{1}^{2}+u_{2}^{2}-u_{3}^{2}-u_{4}^{2}
\end{array}\right),
$$

а оператор $\widehat{w} \equiv-i \hbar \partial / \partial u$.

В этом представлении когерентные состояния (3.12) имеют вид

$$
\begin{aligned}
\mathfrak{H}_{z}= & \frac{\omega}{\pi \hbar}\left(\frac{\sqrt{\omega}\left(u_{1}+i \operatorname{sgn}\left(m_{1}-m_{2}+(l-r)(d-j)\right) u_{2}\right)}{\sqrt{\hbar}}\right)^{\left|m_{1}-m_{2}+(l-r)(d-j)\right|} \times \\
& \times\left(\frac{\sqrt{\omega}\left(u_{3}-i \operatorname{sgn}\left(m_{1}-m_{2}-(l-r) j\right) u_{4}\right)}{\sqrt{\hbar}}\right)^{\left|m_{1}-m_{2}-(l-r) j\right|} \exp \left\{-\frac{\omega|u|^{2}}{2 \hbar}\right\} \times \\
& \times \sum_{j=0}^{d-1-|\Delta m|} \sqrt{\kappa_{j}}\left((-1)^{l} z\right)^{j} L_{\min \left\{r(d-j)-m_{1}-1, l(d-j)-m_{2}-1\right\}}^{\left|m_{1}-m_{2}+(l-r)(d-j)\right|} \\
& \left.\times L_{\min \left\{m_{1}+r j, m_{2}+l j\right\}}^{\left|m_{1}-m_{2}-(l-r) j\right|} \frac{\omega\left(u_{3}^{2}+u_{2}^{2}\right)}{\hbar}\right) \times
\end{aligned}
$$

где $\kappa_{j}$ - коэффициенты (3.8), а $L_{N}^{s}$ - нормированные обобщенные полиномы Лагерра [9]:

$$
L_{N}^{s}(y)=\sum_{j=0}^{N} \frac{\sqrt{N ! \Gamma(N+s+1)}}{(N-j) ! j ! \Gamma(j+s+1)}(-1)^{N-j} y^{j} .
$$

Благодарности. Работа выполнена при поддержке Министерства образования России (грант PD02-1.1-39), а также INTAS (грант 00-257).

\section{Список литературы}

[1] В. П. Маслов. Операторные методы. М.: Наука, 1973.

[2] M. В. Карасев, E. М. Новикова. ТМФ. 2004. Т. 141. № 3. С. 424-454.

[3] М. В. Карасев, Е. М. Новикова. УМН. 1994. Т. 49. № 5. С. 169-170.

[4] М. В. Карасев, Е. М. Новикова. ТМФ. 1996. Т. 108. № 3. С. 339-387.

[5] H. V. McIntosh, A. Cisneros. J. Math. Phys. 1970. V. 11. № 3. P. 896-916; I. Mladenov, V. Tsanov. J. Phys. A. 1987. V. 20. P. 5865-5871; T. Iwai, Y. Uwano. J. Phys. A. 1988. V. 21. P. 4083-4104; I. Mladenov. Ann. Inst. H. Poincare. 1989. V. 50. P. 219-227; A. Yoshioka, K. Ii. J. Math. Phys. 1990. V. 31. № 6. P. 1388-1394.

[6] M. V. Karasev, E. M. Novikova. Adapted connections, Hamilton dynamics, geometric phases and quantization over isotropic submanifolds. In: Coherent Transform, Quantization, and Poisson Geometry. Ed. M. V. Karasev. Providence, RI: AMS Publ., 1998. P. 1-202.

[7] Г. Бейтмен, А. Эрдейи. Высшие трансцендентные функции. Т. 1. М.: Наука, 1973.

[8] Э. Камке. Справочник по обыкновенным дифференциальным уравнениям. М.: Наука, 1971.

[9] Г. Бейтмен, А. Эрдейи. Высшие трансцендентные функции. Т. 2. М.: Наука, 1974.

Поступила в редакцию 12.IV.2004 г. 